\title{
EXISTENCE AND UNIQUENESS OF SOLUTIONS OF SMOLUCHOWSKI'S COAGULATION EQUATION WITH SOURCE TERMS
}

\author{
BY \\ M. SHIRVANI AND H. J. VAN ROESSEL
}

Department of Mathematical Sciences, University of Alberta, Edmonton, Alberta, Canada

\begin{abstract}
We prove the existence and uniqueness of a solution to the Smoluchowski coagulation equation with source terms. The coagulation equation with source terms is potentially useful in applications because one sometimes tries to control coagulation processes by the introduction of particles of various sizes into the system. The existence proof given here differs in style from most other existence proofs in two respects. First, it is not based on a finite-dimensional truncation of the coagulation equation; and secondly, it is achieved with a weaker hypothesis than is usually assumed on the initial data.
\end{abstract}

1. Introduction. Colloidal science is an important area of research both theoretically and experimentally. One of the earliest attempts to understand coagulation, and the first to derive a mathematical model, was Smoluchowski [20, 21]. He made the assumption that collisions are binary, and fluctuations in density are small in order that collisions occur at random. The coagulation equation of Smoluchowski [20, 21]

$$
\frac{d x_{k}}{d t}=\frac{1}{2} \sum_{i+j=k} K_{i j} x_{i} x_{j}-x_{k} \sum_{i=1}^{\infty} K_{k i} x_{i}, \quad x_{k}(0)=c_{k}, \quad k=1,2, \ldots,
$$

where $x_{k}(t)$ is the number (or density) of particles of mass (or type) $k$ at time $t$, models a process where two particles of sizes $i$ and $j$ can coagulate to form a particle of size $i+j$, with a probability proportional to $K_{i j}=K_{j i}>0$.

This infinite system of nonlinear ordinary differential equations and related equations now models colloidal processes in astrophysics [5], polymer chemistry [6], aerosol science [8], and hematology [17]. Theoretical questions concerning existence and/or uniqueness of solutions to (1.1) have been examined by many people. McLeod [15, 16, 14] proved local existence and uniqueness results to (1.1) for the special case of monodisperse initial conditions: $c_{k}=0, k \geq 2$. White [23], Leyvraz and Tschudi [13], and Spouge [22] prove

Received May 15, 2000.

2000 Mathematics Subject Classification. Primary 82D60; Secondary 82Cxx, 34C60.

Research was supported by the Natural Sciences and Engineering Research Council of Canada.

E-mail address: mazi.shirvaniQualberta.ca

E-mail address: henry.vanroessel@ualberta.ca 
existence of a solution, with suitable restrictions on the coagulation kernel $K_{i j}$, but are unable to get uniqueness. Uniqueness, under certain circumstances, was later obtained by Ball and Carr [3] and da Costa [7].

The qualitative nature of solutions has also been studied by many people. The asymptotic behaviour of the Becker-Döring cluster equations, a specialized version of (1.1) with $K_{i j}=0$ if both $i, j$ are greater than 1, was studied by Ball et al. [4]. Ball and Carr [2], and Slemrod [19].

In industrial applications one might want to exercise some control over the coagulation process. For instance, it may be desirable to increase or restrict the limiting number of particles of a particular size. One might attempt to achieve this by the introduction of particles of some prescribed size to enhance the coagulation process to arrive at some desired limiting state. Almost all prior work on Smoluchowski's equation has been either for the case of pure coagulation or coagulation with fragmentation. Little work has been done on Smoluchowski's equation with source terms.

In this article we consider a version of the discrete Smoluchowski coagulation equation (1.1) with nontrivial source terms. As a first step in understanding the effect of source terms we consider pure coagulation with constant coagulation kernel (which may be taken to be $K_{i j}=1$ for all $i, j$ ). The equation we consider is:

$$
\frac{d x_{k}}{d t}=\frac{1}{2} \sum_{i+j=k} x_{i} x_{j}-x_{k} \sum_{i=1}^{\infty} x_{i}+g_{k}, \quad x_{k}(0)=c_{k} . \quad k=1,2, \ldots
$$

where $g_{k}=g_{k}(t)$ is a source term, for all $k$.

For physical reasons the source terms and initial data are assumed to be nommegative. In addition, we do not assume the $g_{k}$ 's to be continuous, since we want to allow for the source terms to be "turned on" or "turned off" at various times. The following basic assumptions will be made throughout:

(A1) $g_{k} \in L_{\text {loc }}^{1}[0, T)$, where $T \leq \infty$, for $k=1,2, \ldots$.

(A2) $c_{k} \geq 0$ and $g_{k}(t) \geq 0$ for a.c. $t \in[0, T)$ for $k=1.2 \ldots$

In papers dealing with Smoluchowski's equation (1.1) without source terms, the primary concern is existence of density-conserving solutions. This requires that the first moments of the initial conditions be finite, that is, $\sum_{k=1}^{\infty} k c_{k}<\infty$. It has been demonstrated $[3,11,12,13,18]$ that for some rate coefficients, density conservation breaks down at some finite time $t=t_{g} \geq 0$. This is physically interpreted as corresponding to the occurrence of a phase transition in the system called gelation. With the presence of source terms, one would not expect density-conserving solutions at all. In light of this, we make the weaker assumption

(A3) $\sum_{k=1}^{\infty} c_{k}<\infty$.

Finally, we require that the total source of all particles not be too large; that is, we assume

(A4) $\sum_{k=1}^{\infty} g_{k} \in L_{\text {loc }}^{1}[0, T)$.

Because of the discontinuous right-hand side of (1.2), it is necessary to say what we mean by a solution to the equation. By a solution of Eq. (1.2) we mean a solution in the sense of Carathéodory (see [10]): i.e.. a solution that is absolutely continuous on compact 
subsets of $[0, T)$ and satisfies the differential equation (1.2) almost everywhere. The main theorem that we will prove is the following:

Theorem 1.1 (Existence and Uniqueness). If assumptions (A1), (A2), (A3), and (A4) hold, then Eq. (1.2) has a unique nonnegative solution on $[0, T)$.

The procedure most commonly followed to prove existence to (1.1) consists in taking finite-dimensional truncations $x_{k}^{(n)}$ of the system, and then showing that these converge to $x_{k}$ in some sense. The approach taken here is somewhat different. Because of the presence of an infinite sum, representing the total number of particles, in each equation in (1.2), it is convenient to consider two auxiliary equations. The first is

$$
X^{\prime}=-\frac{1}{2} X^{2}+g, \quad X(0)=c,
$$

which is obtained by formally summing the system (1.2). The second is the linear system

$$
\frac{d y_{k}}{d t}+X y_{k}=\frac{1}{2} \sum_{i+j=k} y_{i} y_{j}+g_{k}, \quad y_{k}(0)=c_{k}, \quad k=1,2, \ldots
$$

The proof of Theorem 1.1 will be given in two stages: existence of a solution will be proven in Sec. 2, while uniqueness will be proven in Sec. 3. Some properties of the solution will be exhibited in Sec. 4 , and some examples given in Sec. 5 .

2. Proof of existence. To begin we examine the Riccati equation (1.3). In particular, we show that solutions to (1.3) are nonnegative. We quote the following differential inequality, which will prove useful.

Lemma 2.1 (see Hale [10], p. 31). Let $\omega(t, u)$ be continuous on an open connected set $\Omega \subset \mathbb{R}^{2}$. If $u$ is a solution of $u^{\prime}(t)=\omega(t, u(t))$ for $t \in[a, b]$ and $v$ satisfies $v^{\prime}(t) \leq \omega(t, v(t))$ on $[a, b)$ with $v(a) \leq u(a)$, then $v(t) \leq u(t)$ for $t \in[a, b]$.

The following lemma will prove to be more than we actually need.

LEMmA 2.2. Let $f$ be a continuous function that satisfies $f^{\prime} \geq-\delta f^{2}$ for almost all $t \in[0, T)$, where $\delta>0$ and $0<T \leq \infty$. Then the following are the only possibilities:

1. $f$ has no zeros on $[0, T)$;

2. $f=0$ on an interval $[a, b]$, with $0 \leq a \leq b<T$ where $f<0$ on $[0, a)$ and $f>0$ on $(b, T)$;

3. $f<0$ on $[0, a)$, and $f=0$ on $[a, T)$.

Proof. Consider the inequality on the subinterval $[\alpha, T)$ for some $\alpha \in[0, T)$. Let $v=-f$. Then $v$ satisfies $v^{\prime} \leq \delta v^{2}$ on $[\alpha, T)$. Using Lemma 2.1 with $\omega(t, x)=\delta x^{2}$ and $v(\alpha)=u(\alpha)$ we get

$$
v(t) \leq \frac{v(\alpha)}{1-\delta v(\alpha)(t-\alpha)} \quad \text { for } t \in[\alpha, T)
$$

which leads to

$$
f(t) \geq \frac{f(\alpha)}{1+\delta f(\alpha)(t-\alpha)} \quad \text { for } t \in[\alpha, T)
$$


Let $S=\{t \in[0, T): f(t)=0\}$ be the set of zeros of $f$. If $S=\varnothing$, then we have case 1. If $S \neq \varnothing$ set $a:=\inf S$. Then continuity of $f$ implies that $a \in S$ so that $f(a)=0$. Inequality (2.2) then implies that $f(t) \geq 0$ on $[a, T)$. For $\alpha<a$ it follows that $f(\alpha)<0$ since $f(\alpha)=0$ would contradict the definition of $a$ and $f(\alpha)>0$ would imply, via (2.2), that $f(a)>0$.

Now set $b:=\sup S$. There are two cases to consider: $b<T$ and $b=T$. If $b<T$, then continuity of $f$ implies that $b \in S$ so that $f(b)=0$. If $b>a$ choose an arbitrary $\alpha \in(a, b)$. Then $f(t) \geq 0$ on $[a, T)$ implies that $f(\alpha) \geq 0$. On the other hand, $f(\alpha)>0$ would imply that $f(b)>0$, which is a contradiction since $b \in S$. Therefore $f(\alpha)=0$, and hence $S=[a, b]$. Case 2 results.

If $b=T$, then $S=[a, T)$ and case 3 results.

The main property of $X$ now follows as an immediate consequence of Lemma 2.2.

Corollary 2.3. If (i) $X$ is a solution of (1.3) on $[0, T)$ and (ii) $c \geq 0$, then $X \geq 0$ on $[0, T)$.

Having established the nonnegativity of $X$, we consider the sequence of equations (1.4) on the subinterval $[\alpha, T)$, for some $\alpha \geq 0$. Assumption (A1) guarantees that this linear system has a unique solution on $[\alpha, T)$ given by

$$
y_{k}(t)=e^{-\mu(t)} y_{k}(\alpha)+e^{-\mu(t)} \int_{\alpha}^{t} e^{\mu(\tau)}\left(\frac{1}{2} \sum_{i+j=k} y_{i}(\tau) y_{j}(\tau)+g_{k}(\tau)\right) d \tau,
$$

where

$$
\mu(t):=\int_{\alpha}^{t} X(\tau) d \tau
$$

LEMma 2.4. Suppose assumptions (A1) through (A4) hold and $y_{k}(\alpha) \geq 0$ for all $k$ and $\sum_{k=1}^{\infty} y_{k}(\alpha)<\infty$. Then there exists $\beta>\alpha$ such that $\sum_{k=1}^{\infty} y_{k}(t)$ converges uniformly on $[\alpha, \beta]$.

Proof. Define the following:

$$
\begin{aligned}
Y(\alpha) & :=\sum_{k=1}^{\infty} y_{k}(\alpha), \quad m(t):=\sup _{\alpha \leq s \leq t} e^{-\mu(s)} \int_{\alpha}^{s} e^{\mu(\tau)} d \tau, \\
g(t) & :=\sum_{k=1}^{\infty} g_{k}(t), \quad d_{k}(t):=\sup _{\alpha \leq s \leq t} y_{k}(s), \\
p_{k}(t) & :=\sup _{\alpha \leq s \leq t}\left(y_{k}(\alpha) e^{-\mu(s)}+e^{-\mu(s)} \int_{\alpha}^{s} e^{\mu(\tau)} g_{k}(\tau) d \tau\right) .
\end{aligned}
$$

Since $\mu$ is a nonnegative, nondecreasing function (which follows from Corollary 2.3) and $y_{k}(\alpha)$ and $g_{k}$ are nonnegative, we may obtain an upper bound on $p_{k}$ by replacing $e^{-\mu(s)}$ by 1 and $e^{\mu(\tau)}$ by $e^{\mu(t)}$. This yields

$$
p_{k}(t) \leq y_{k}(\alpha)+e^{\mu(t)} \int_{\alpha}^{t} g_{k}(\tau) d \tau
$$


Summing from 1 to $n$, we obtain

$$
\sum_{k=1}^{n} p_{k}(t) \leq \sum_{k=1}^{n} y_{k}(\alpha)+e^{\mu(t)} \int_{\alpha}^{t} \sum_{k=1}^{n} g_{k}(\tau) d \tau \leq Y(\alpha)+e^{\mu(t)} \int_{\alpha}^{t} g(\tau) d \tau .
$$

Letting $n \rightarrow \infty$, we find that

$$
\sum_{k=1}^{\infty} p_{k}(t) \leq Y(\alpha)+e^{\mu(t)} \int_{\alpha}^{t} g(\tau) d \tau<\infty
$$

Next, the right-hand side of (2.3) can be majorized by

$$
p_{k}(t)+\frac{1}{2} \sup _{\alpha \leq s \leq t} \sum_{i+j=k} e^{-\mu(s)} \int_{\alpha}^{s} e^{\mu(\tau)} y_{i}(\tau) y_{j}(\tau) d \tau .
$$

Upon switching the sum and the sup and replacing $y_{i}$ and $y_{j}$ by $d_{i}$ and $d_{j}$, we obtain

$$
d_{k}(t) \leq p_{k}(t)+\frac{m(t)}{2} \sum_{i+j=k} d_{i}(t) d_{j}(t), \quad k \geq 1
$$

Define a new sequence $e_{k}$ by $e_{1}(t)=d_{1}(t)$ and

$$
e_{k}(t)=p_{k}(t)+\frac{m(t)}{2} \sum_{i+j=k} e_{i}(t) e_{j}(t), \quad k \geq 2 .
$$

A simple inductive argument shows that $d_{k} \leq e_{k}$ for all $k$. In terms of the generating functions

$$
E(t, z):=\sum_{k=1}^{\infty} e_{k}(t) z^{k}, \quad P(t, z):=\sum_{k=1}^{\infty} p_{k}(t) z^{k}
$$

Eq. (2.5) may be written as

$$
E(t, z)=P(t, z)+\frac{m(t)}{2} E(t, z)^{2}
$$

whose solution is

$$
E=\frac{m}{2}(1-\sqrt{1-2 m P}) .
$$

Since $\sum_{k=1}^{\infty} p_{k}(t)<\infty$, while $m(t) \rightarrow 0$ as $t \rightarrow \alpha^{+}$, it is clear that $\sum_{k=1}^{\infty} p_{k}(t)<\frac{1}{2 m(t)}$ is satisfied for all $t \geq \alpha$ but sufficiently near $\alpha$ (i.e., for all $t \in[\alpha, \beta]$ for some $\beta>\alpha$ ). Then for $|z| \leq 1$ we have

$$
|2 m P(t, z)| \leq 2 m \sum_{k=1}^{\infty} p_{k}(t)|z|^{k} \leq \sum_{k=1}^{\infty} p_{k}(t)<1 .
$$

It follows that $\sqrt{1-2 m(t) P(t, z)}$ has radius of convergence (as a function of $z$ ) greater than 1. In particular, $\sum_{k=1}^{\infty} e_{k}(t)=E(t, 1)<\infty$. For $t \in[\alpha, \beta]$ the series $\sum_{k=1}^{\infty} y_{k}(t)$ is therefore majorized by $\sum_{k=1}^{\infty} e_{k}(\beta)$. By the M-test, $\sum_{k=1}^{\infty} y_{k}(t)$ converges uniformly on $[\alpha, \beta]$.

Corollary 2.5. The series $\sum_{k=1}^{\infty} y_{k}(t)$ converges uniformly on $[0, \beta]$ for some $\beta>0$.

Define $Y(t):=\sum_{k=1}^{\infty} y_{k}(t)$. Then we have the following: 
LEMMA 2.6. If assumptions (A1) through (A4) hold, then there exists $\beta>0$ such that $Y=X$ on $[0, \beta]$.

Proof. Setting $Y_{n}(t):=\sum_{k=1}^{n} y_{k}(t)$ and $G_{n}(t):=\sum_{k=1}^{n} g_{k}(t)$ and letting $\alpha=0$ in (2.3), we obtain

$$
Y_{n}(t)=e^{-\mu(t)}\left(Y_{n}(0)+\frac{1}{2} \int_{0}^{t} e^{\mu(\tau)} \sum_{k=1}^{n} \sum_{i+j=k} y_{i}(\tau) y_{j}(\tau) d \tau+\int_{0}^{t} e^{\mu(\tau)} G_{n}(\tau) d \tau\right)
$$

Since the $y_{k}$ 's are continuous and $Y(t)=\sum_{k=1}^{\infty} y_{k}(t)$ converges uniformly on $[0, \beta]$, it follows that $Y$ is also continuous and hence bounded on $[0, \beta]$. Therefore,

$$
\sum_{k=1}^{n} \sum_{i+j=k} y_{i} y_{j} \leq Y^{2}
$$

is bounded on $[0, \beta]$. Letting $n \rightarrow \infty$, and using the Dominated Convergence Theorem, we get

$$
Y(t)=e^{-\mu(t)}\left(Y(0)+\frac{1}{2} \int_{0}^{t} e^{\mu(\tau)} Y^{2}(\tau) d \tau+\int_{0}^{t} e^{\mu(\tau)} g(\tau) d \tau\right) .
$$

Thus $Y$ is absolutely continuous on $[0, \beta]$, and hence differentiable almost everywhere. Differentiating, we get

$$
Y^{\prime}(t)=-X(t) Y(t)+\frac{1}{2} Y^{2}(t)+g(t) .
$$

Using Eq. (1.3) to eliminate $g$ results in

$$
Y^{\prime}(t)=-X(t) Y(t)+\frac{1}{2} Y^{2}(t)+X^{\prime}(t)-\frac{1}{2} X^{2}(t)
$$

which can be rewritten as $(Y-X)^{\prime}=\frac{1}{2}(Y-X)^{2}$. The common initial condition $Y(0)=$ $X(0)=c$ gives the result.

We can extend the previous result to the entire interval $[0, T)$.

Lemma 2.7. If assumptions (A1) through (A4) hold, then $Y=X$ on $[0, T)$.

Proof. Let $S=\left\{\beta \in[0, T): \sum_{k=1}^{\infty} y_{k}\right.$ converges uniformly on $\left.[0, \beta]\right\}$ and define $\tau:=\sup S$. Then $\tau \notin S$ since $\tau \in S$ implies, using Lemma 2.4, that the series converges uniformly on the interval $[\tau, \tau+\varepsilon]$, which contradicts the definition of $\tau$.

Then $Y(t)$ exists for all $t \in[0, \tau)$ and $Y=X$ on $[0, \tau)$. Assumption (A1) implies that $y_{k}(t) \geq 0$ for all $t \in[0, T)$ and all $k$. Therefore, $\left\{Y_{n}(t)\right\}_{n=1}^{\infty}$ is an increasing sequence that satisfies

$$
Y_{n}(t) \leq Y(t)=X(t) \text { for } t \in[0, \tau) \subseteq[0, T) .
$$

There are two possibilities: $\tau<T$ or $\tau=T$. Suppose $\tau<T$. Then since $Y_{n}$ and $X$ are continuous on $[0, T)$, we have $Y_{n}(\tau) \leq X(\tau)$. Letting $n \rightarrow \infty$ we get $Y(\tau)=$ $\lim _{n \rightarrow \infty} Y_{n}(\tau)=X(\tau)$, which implies that $\tau \in S$. This is a contradiction, and therefore $\tau=T$.

Existence of a solution now follows easily. Lemma 2.7 implies that $X=\sum_{k=1}^{\infty} y_{k}$ for all $t \in[0, T)$. Therefore, Eq. (1.4) is identical to Eq. (1.2), which is another way of saying that the $\left\{y_{k}\right\}$ are a solution to (1.2). 
3. Proof of uniqueness. Suppose $\left\{x_{k}\right\}$ is a solution to (1.2) on $[0, T)$. Then the sum $P(t):=\sum_{k=1}^{\infty} x_{k}(t)$ converges for a.e. $t \in[0, T)$ and Eq. (1.2) becomes

$$
x_{k}^{\prime}=\frac{1}{2} \sum_{i+j=k} x_{i} x_{j}-P x_{k}+g_{k} .
$$

LEMma 3.1. Assume that (A1) through (A4) hold. If $\left\{x_{k}\right\}$ is any nonnegative solution to Eq. (1.2) on $[0, T)$, then $P$ is bounded on an interval $[0, a]$ for some $0<a<T$.

Proof. Choose $\tau \in[0, T)$, any $b>c+\int_{0}^{\tau} g(s) d s$, and let

$$
a<\min \left\{\frac{2}{b^{2}}\left(b-c-\int_{0}^{\tau} g(s) d s\right), \tau\right\} .
$$

Let $P_{n}(t)=\sum_{k=1}^{n} x_{k}(t)$. Since $P=\sum_{k=1}^{\infty} x_{k} \geq 0$, we have

$$
x_{k}^{\prime}=\frac{1}{2} \sum_{i+j=k} x_{i} x_{j}-P x_{k}+g_{k} \leq \frac{1}{2} \sum_{i+j=k} x_{i} x_{j}+g_{k} .
$$

Therefore,

$$
x_{k}(t) \leq x_{k}(0)+\frac{1}{2} \int_{0}^{t} \sum_{i+j=k} x_{i}(s) x_{j}(s) d s+\int_{0}^{t} g_{k}(s) d s
$$

for all $t \in[0, a]$. Summing from 1 to $n$ we obtain

$$
P_{n}(t) \leq c+\frac{1}{2} \int_{0}^{t} \sum_{k=1}^{n} \sum_{i+j=k} x_{i}(s) x_{j}(s) d s+\int_{0}^{t} g(s) d s .
$$

The double sum is at most $P_{n-1}^{2}$. So finally

$$
P_{n}(t) \leq c+\frac{1}{2} \int_{0}^{a} P_{n-1}^{2}(s) d s+\int_{0}^{\tau} g(s) d s \quad \text { for all } t \in[0, a] .
$$

In particular, on $[0, a]$ we have $P_{1} \leq c+\int_{0}^{\tau} g(s) d s<b$ by the choice of $b$. If $P_{n-1}<b$ on $[0, a]$, then (3.2) implies that

$$
P_{n}(t) \leq c+\frac{1}{2} \int_{0}^{a} b^{2} d s+\int_{0}^{a} g(s) d s=c+\frac{1}{2} a b^{2}+\int_{0}^{\tau} g(s) d s<b
$$

by the way $a$ was chosen. By induction, every $P_{n}<b$; so $P$ is also bounded on $[0, a]$.

LEmma 3.2. Assume that (A1) through (A4) hold and that $\left\{x_{k}\right\}$ is any nonnegative solution to (1.2). Then $P=X$ on $[0, a]$ for some $a<T$.

Proof. From Lemma 3.1 it follows that $P$ is bounded on $[0, a]$ for some $a<T$; so $P$ is integrable on $[0, a]$. Let $M(t)=\int_{0}^{t} P(\tau) d \tau$ for all $t \in[0, a]$. Then the $x_{k}$ satisfy

$$
x_{k}(t)=e^{-M(t)}\left(x_{k}(0)+\frac{1}{2} \int_{0}^{t} e^{M(\tau)} \sum_{i+j=k} x_{i}(\tau) x_{j}(\tau) d \tau+\int_{0}^{t} e^{M(\tau)} g_{k}(\tau) d \tau\right)
$$

for all $t \in[0, a]$. Summing from 1 to $n$ yields

$$
P_{n}(t)=e^{-M(t)}\left[P_{n}(0)+\frac{1}{2} \int_{0}^{t} e^{M(\tau)} \sum_{k=1}^{n} \sum_{i+j=k} x_{i}(\tau) x_{j}(\tau) d \tau+\int_{0}^{t} e^{M(\tau)} G_{n}(\tau) d \tau\right]
$$


Since $\sum_{k=1}^{n} \sum_{i+j=k} x_{i} x_{j} \leq P^{2}$ is bounded, and hence integrable on $[0, a]$, letting $n \rightarrow \infty$ and using the Dominated Convergence Theorem yields

$$
P(t)=e^{-M(t)}\left(P(0)+\frac{1}{2} \int_{0}^{t} e^{M(\tau)} P^{2}(\tau) d \tau+\int_{0}^{t} e^{M(\tau)} g(\tau) d \tau\right) .
$$

Thus $P$ is absolutely continuous on $[0, a]$, and hence differentiable almost everywhere. Differentiating, we get

$$
P^{\prime}(t)=-\frac{1}{2} P^{2}(t)+g(t) .
$$

Since $P$ satisfies the same Riccati equation as $X$ almost everywhere, $P(0)=X(0)$, and $P$ and $X$ are everywhere continuous, it follows that $P=X$, as required.

We can extend the previous result to the entire interval $[0, T)$.

Lemma 3.3. If assumptions (A1) through (A4) hold, then $P=X$ on $[0, T)$.

Proof. We know from Lemma 3.2 that $P=X$ on $[0, \alpha]$ for some $\alpha<T$. Therefore, Equations (2.3) and (3.3) are equivalent on $[0, \alpha]$. So we may apply Lemma 2.4 to (3.3) to conclude that if $P(\alpha)$ is bounded, then $P$ is bounded on $[\alpha, \beta]$ for some $\beta>\alpha$.

Let $S=\{\beta \in[0, T): P$ is bounded on $[0, \beta]\}$. Set $\tau:=\sup S$. Then $\tau \notin S$ since $\tau \in S$ implies, using Lemma 2.4 , that $P$ is bounded on the interval $[\tau, \tau+\varepsilon]$, which contradicts the definition of $\tau$.

By Lemma 3.2 , we know that $P=X$ on every closed subinterval of $[0, \tau)$, and so $P=X$ on $[0, \tau)$. There are two possibilities: either $\tau<T$ or $\tau=T$.

Suppose $\tau<T$. Then for any $n$ and any $t<\tau$ we have $P_{n}(t) \leq P(t)=X(t)$. Letting $t \rightarrow \tau^{-}$we obtain $P_{n}(\tau) \leq X(\tau)$, whence $P(\tau)=\lim P_{n}(\tau)$ is also finite. So $\tau \in S$, which is a contradiction. Therefore $\tau=T$.

By Lemmas 3.3 and 3.2 we know that $P=X$ throughout the interval $[0, T)$. Equation (1.4) therefore reduces to (1.2).

\section{Properties of the solution.}

Lemma 4.1. If $g(t)>0$ for almost all $t \in(0, \tau)$ for some $\tau \leq T$, then $X(t)>0$ for all $t \in(0, \tau)$.

Proof. $X$ satisfies one of the three conditions of Corollary 2.3. But if $X=0$ on an interval, then $g=X^{\prime}+\frac{1}{2} X^{2}=0$ for almost all $t$ in the same interval, which contradicts the positivity of $g$. The result follows.

Lemma 4.2. Let $K$ be a constant, and $f$ a function such that $f^{\prime} \leq-\delta f^{2}+K$ on $[0, T)$ where $0<T \leq \infty$. Then $f$ is bounded above on $[0, T)$.

Proof. Let $b>\sqrt{K / \delta}$ and $n>0$ be fixed positive constants, and consider the set

$$
S_{n}=\{t: t \in[0, T) \text { and } f(t) \geq b+n\} .
$$

Suppose that every $S_{n}$ is nonempty (which is equivalent to $f$ not being bounded above), and let $t_{n}=\inf S_{n} \geq 0$. Then $f\left(t_{n}\right) \geq b+n>b$ by continuity; so $f^{\prime}\left(t_{n}\right) \leq-\delta f\left(t_{n}\right)^{2}+$ $K<0$. If $t_{n}>0$, then $f$ is decreasing at $t_{n}$, whence there exists $t \in\left(0, t_{n}\right)$ such that 
$f(t)>f\left(t_{n}\right) \geq b+n$. Thus $t \in S_{n}$, contradicting the definition of $t_{n}$. Therefore, $t_{n}=0$; so $f(0) \geq b+n$ for every positive $n$, which is absurd. the result follows.

Theorem 4.3. Suppose assumptions (A1) through (A4) hold. If $g(T)=\lim _{t \rightarrow T^{-}} g(t)$ exists, then so does $X(T)$.

Proof. Lemma 4.2 implies that $X$ is bounded. In particular, if $X$ is eventually monotonic (i.e., either nonincreasing or nondecreasing for all $t$ sufficiently near $T$ ), then the result is true. Assume, therefore, that $X$ is not eventually monotonic so that $X$ has an infinite number of extreme values in $[0, T)$. Let $\left\{t_{n}\right\}_{n=1}^{\infty} \subset[0, T)$ with $t_{n} \rightarrow T$ as $n \rightarrow \infty$ be the sequence of points at which the local extrema of $X$ occur. If $X$ has a local minimum at $t_{n}$, then $X$ will have a local maximum at $t_{n+1}$ so that

$$
X\left(t_{n}\right) \leq X(t) \leq X\left(t_{n+1}\right) \text { for } t \in\left[t_{n}, t_{n+1}\right] .
$$

Since $X^{\prime}\left(t_{n}\right)=0$, we get $\lim _{n \rightarrow \infty} X\left(t_{n}\right)=\lim _{n \rightarrow \infty} \sqrt{2 g\left(t_{n}\right)}=\sqrt{2 g(T)}$, from which it follows that

$$
X(T)=\lim _{t \rightarrow T^{-}} X(t)=\sqrt{2 g(T)}
$$

The example $X(t)=4+\sin t$ and $g(t)=\cos t+4 \sin t+8+\frac{1}{2} \sin ^{2} t$ on $[0, \infty)$ shows that the conclusion of Theorem 4.3 does not necessarily hold if $g$ does not have a limit as $t \rightarrow \infty$. (Note that $g \geq-\sqrt{1+4^{2}}+8>0$ for all $t$.)

In some industrial applications, it is desirable to have a system without too many large particles. Ideally, we would like to have $x_{k}(\infty)=0$ for all $k \geq 2$. This is, however, impossible with a steady-state input as the following theorem demonstrates.

Theorem 4.4. Suppose that assumptions (A1) through (A4) hold with $T=\infty$. In addition, suppose that $g(\infty)$ exists and $g_{k}(\infty)$ exist for all $k$. Then $x_{k}(\infty)$ exist for all $k$ and are given by

$$
x_{k}(\infty)= \begin{cases}0 & \text { if } g(\infty)=0 \\ \frac{1}{\sqrt{2 g(\infty)}}\left[\frac{1}{2} \sum_{i+j=k} x_{i}(\infty) x_{j}(\infty)+g_{k}(\infty)\right] & \text { if } g(\infty)>0\end{cases}
$$

Proof. First consider the case $g(\infty)=0$. Since $X=\sum_{k=1}^{\infty} x_{k}$ it follows that $0 \leq$ $x_{k}(t) \leq X(t)$ for all $t$. Letting $t \rightarrow \infty$ and using Theorem 4.3 yields $0 \leq x_{k}(\infty) \leq$ $X(\infty)=\sqrt{2 g(\infty)}=0$.

Now suppose $g(\infty)>0$. Then $X(\infty)=\sqrt{2 g(\infty)}>0$, which implies that $\mu(\infty)=$ $\int_{0}^{\infty} X(\tau) d \tau=\infty$. The solution to $(1.2)$ with $k=1$ is

$$
x_{1}(t)=e^{-\mu(t)}\left(c_{1}+L_{1}(t)\right)
$$

where

$$
L_{k}(t):=\int_{0}^{t} e^{\mu(\tau)} g_{k}(\tau) d \tau, \quad k=1,2, \ldots
$$


There are two possibilities for $L_{1}(\infty)$ : either $L_{1}(\infty)<\infty$ or $L_{1}(\infty)=\infty$. If $L_{1}(\infty)<\infty$, then $g_{1}(\infty)=0$ and

$$
x_{1}(\infty)=\lim _{t \rightarrow \infty} e^{-\mu(t)}\left(c_{1}+L_{1}(t)\right)=0=\frac{g_{1}(\infty)}{\sqrt{2 g(\infty)}} .
$$

If $L_{1}(\infty)=\infty$, then with the aid of l'Hôpital's rule we get

$$
x_{1}(\infty)=\lim _{t \rightarrow \infty} \frac{c_{1}+L_{1}(t)}{e^{\mu(t)}}=\lim _{t \rightarrow \infty} \frac{e^{\mu(t)} g_{1}(t)}{X(t) e^{\mu(t)}}=\frac{g_{1}(\infty)}{X(\infty)}=\frac{g_{1}(\infty)}{\sqrt{2 g(\infty)}} .
$$

In either case we get $x_{1}(\infty)=\frac{g_{1}(\infty)}{\sqrt{2 g(\infty)}}$. Inductively, assuming that $x_{i}(\infty)$ exists for all $i<k$, the same argument leads to

$$
x_{k}(\infty)=\lim _{t \rightarrow \infty} x_{k}(t)=\frac{1}{\sqrt{2 g(\infty)}}\left[\frac{1}{2} \sum_{i+j=k} x_{i}(\infty) x_{j}(\infty)+g_{k}(\infty)\right] .
$$

5. Examples. We finish off with a couple of examples. There is also a useful formal method of treating the system (1.4). Consider the generating functions

$$
f(t, z)=\sum_{k=1}^{\infty} x_{k}(t) z^{k} \quad \text { and } \quad G(t, z)=\sum_{k=1}^{\infty} g_{k}(t) z^{k} .
$$

Then formal summation of (1.4) yields

$$
\frac{\partial f}{\partial t}+X f=\frac{1}{2} f^{2}+G, \quad f(0, z)=c
$$

Letting $t \rightarrow \infty$ yields

$$
X(\infty) f(\infty, z)=\frac{1}{2} f^{2}(\infty, z)+G(\infty, z)
$$

whose solution is

$$
f(\infty, z)=X(\infty)-\sqrt{X^{2}(\infty)-2 G(\infty, z)}=\sqrt{2 g(\infty)}-\sqrt{2[g(\infty)-G(\infty, z)]} .
$$

EXAmple 5.1. Consider the case where all the source terms decay to zero except the source term for the smallest particles. That is, assume that $g_{k}(\infty)=0$ for all $k \geq 2$, and $g_{1}(\infty)=2 b^{2}$. Then $g(\infty)=2 b^{2}$ and Eq. (5.3) implies that $\sum_{k=1}^{\infty} x_{k}(\infty) z^{k}=$ $2 b(1-\sqrt{1-z})$, which results in

$$
x_{1}(\infty)=b \quad \text { and } \quad x_{i}(\infty)=\frac{(2 i-3) !}{i 2^{2 i-3}(i-1) !(i-2) !} b, \quad \text { for } i \geq 2 .
$$

See [9] for this formula.

EXAMPLE 5.2. Consider the case with zero initial conditions and a single source term. Assume that $c=0$ and $g(t)=g_{1}(t)=2\left(1+t^{2}\right)^{-2}$ for all $t$. Then (5.2) becomes

$$
\frac{\partial f}{\partial t}+\frac{25}{1+t^{2}} f=\frac{1}{2} f^{2}+\frac{2 z}{\left(1+t^{2}\right)^{2}}, \quad f(0, z)=0 .
$$

The solution of (5.4) is

$$
f(t, z)=\frac{2 \sqrt{z}}{1+t^{2}} \tan \left(\sqrt{z} \tan ^{-1}(t)\right)
$$


By [1], 4.3.67, we have $\tan (\zeta)=\zeta \sum_{i=0}^{\infty} \alpha_{i} \zeta^{i}$, where

$$
\alpha_{i}=\frac{(-1)^{i} 2^{2 i+2}\left(2^{2 i+2}-1\right) B_{2 i}}{(2 i+2) !} .
$$

Therefore, $f(t, z)=2 \tau\left(1+t^{2}\right)^{-1} \sum_{i=0}^{\infty} \alpha_{i} \arctan ^{2 i}(t) \zeta^{i}$. Using the usual estimate for the Bernoulli number $B_{2 i}([1], 23.1 .15)$, we find that

$$
\alpha_{i-1}=2(-1)^{i-1} \beta_{i}\left(\frac{2}{\pi}\right)^{2 i}\left(1-4^{-i}\right)
$$

where $1<\beta_{i}<\left(1-2^{-2 i+1}\right)^{-1}$. Thus

$$
x_{k}(t)=\frac{4}{1+t^{2}} \beta_{k}\left(\frac{2}{\pi}\right)^{2 k}\left(1-4^{-k}\right) \arctan ^{2 k-1}(t) .
$$

\section{REFERENCES}

[1] Milton Abramowitz and Irene A. Stegun, Handbook of Mathematical Functions, Dover, 1965

[2] J. M. Ball and J. Carr, Asymptotic behaviour of solutions to the Becker-Döring cluster equations for arbitrary initial data, Proc. Roy. Soc. Edinburgh 108A, 109-116 (1988)

[3] J. M. Ball and J. Carr, The discrete coagulation-fragmentation equations: Existence, uniqueness, and density conservation, J. Stat. Phys. 61, 203-234 (1990)

[4] J. M. Ball, J. Carr, and O. Penrose, The Becker-Döring cluster equations: Basic properties and asymptotic behaviour of solutions, Comm. Math. Phys. 104, 657-692 (1986)

[5] J. D. Barrow, Coagulation with fragmentation, J. Phys. A 14, 729-733 (1981)

[6] R. J. Cohen and G. B. Benderek, Equilibrium and kinetic theory of sol-gel transition, J. Chem. Phys. 86, 3696-3714 (1982)

[7] F. P. da Costa, Existence and uniqueness of density conserving solutions to the coagulationfragmentation equations with strong fragmentation, J. Math. Anal. Appl. 192, 892-914 (1995)

[8] R. L. Drake, A general mathematical survey of the coagulation equation, Topics in Current Aerosol Research 3 (Part 2) (G. Hidy and J. R. Brock, eds.), Pergamon Press, 1972

[9] P. B. Dubovskii, Convergence of the solutions of the coagulation equation with a source to an equilibrium state, Differential Equations 31, 635-640 (1995)

[10] Jack K. Hale, Ordinary Differential Equations, second edition, Krieger Publishing Co., Huntington, NY, 1980

[11] E. M. Hendriks, M. H. Ernst, and R. M. Ziff, Coagulation equations with gelation, J. Stat. Phys. 31, 519-563 (1983)

[12] F. Leyvraz, Existence and properties of post-gel solutions for the kinetic equations of coagulation, J. Phys. A: Math. Gen. 16, 2861-2873 (1983)

[13] F. Leyvraz and H. R. Tschudi, Singularities in kinetics of coagulation processes, J. Phys. A: Math. Gen. 14, 3389-3405 (1981)

[14] J. B. McLeod, On a recurrence formula in differential equations, Quart. J. Math. Oxford Ser. (2) 13, 283-284 (1962)

[15] J. B. McLeod, On an infinite set of non-linear differential equations, Quart J. Math. Oxford Ser. (2) 13, 119-128 (1962)

[16] J. B. McLeod, On an infinite set of non-linear differential equations (II), Quart. J. Math. Oxford Ser. (2) 13, 193-205 (1962)

[17] R. W. Samsel and A. S. Perelson, Kinetics of rouleaux formation, Biophys. J. 37, 493-514 (1982)

[18] M. Shirvani and H. J. Van Roessel, The mass-conserving solutions of Smoluchowski's coagulation equation: The general bilinear kernel, Z. Angew. Math. Phys. 43, 526-535 (1992)

[19] M. Slemrod, Trend to equilibrium in the Becker-Döring cluster equations, Nonlinearity 2, 429-443 (1989) 
[20] M. von Smoluchowski, Drei Vorträge über Diffusion, Brownsche Bewegung und Koagulation von Kolloidteilchen, Z. Phys. 17, 557-585 (1916)

[21] M. von Smoluchowski, Versuch einer mathematischen Theorie der Koagulationskinetik kolloider Lösungen, Z. Phys. Chem. 92, 124-168 (1917)

[22] John L. Spouge, An existence theorem for the discrete coagulation-fragmentation equations, Math. Proc. Cambridge Philos. Soc. 96, 351-357 (1984)

[23] W. H. White, A global existence theorem for Smoluchowski's coagulation equations, Proc. Amer. Math. Soc. 80, 273-276 (1980) 